\title{
Separable Control Lyapunov Functions with Application to Prostheses
}

\author{
Rachel Gehlhar and Aaron D. Ames, Senior Member, IEEE
}

\begin{abstract}
This paper extends bipedal trajectory tracking methods to prostheses to enable construction of a class of model-dependent prosthesis controllers using locally available sensor information. The rapidly exponentially stabilizing control Lyapunov functions (RES-CLFs) developed for bipedal robots guarantee stability of the hybrid zero dynamics in the presence of impacts that occur in walking. These methods cannot be directly applied to prostheses because of the unknown human dynamics. We overcome this challenge with two RES-CLFs, one for the prosthesis subsystem and another for the remaining human system. Further, we outline a method to construct these RES-CLFs for this type of separable system by first constructing separable CLFs for partially feedback linearizable systems. This work develops a class of separable subsystem controllers that rely only on local information but provide formal guarantees of stability for the full hybrid system with zero dynamics.
\end{abstract} ics

Index Terms-Lyapunov methods, nonlinear systems, prosthet-

\section{INTRODUCTION}

$\mathbf{P}$ OWERED prostheses commonly use impedance control [1] which is highly heuristic in requiring hand tuning and yields no formal guarantees of stability or optimality. Inspired by bipedal control methods, researchers applied bipedal trajectory generation methods to prostheses [2], [3], but cannot translate bipedal model-based trajectory tracking methods due to unknown human dynamics. Inclusion of the model in the control problem allows inputs to be chosen to satisfy constraints on the physical system [4] and lowers dependence on high-gain PD control by using a feedforward term. Also, through consideration of the nonlinear dynamics, controllers can establish formal guarantees on the stability of the system [5], [6]. Researchers [7], [8] constructed feedback linearizing controllers for prostheses in simulation using the interaction force between the human and prosthesis, demonstrating how to treat subsystems separately for one specific controller. We extend upon this work by developing a class of separable model-dependent controllers.

In developing a class of controllers for bipedal robots, researchers looked to establish stability given the impacts and zero dynamics present in walking. Using a RES-CLF, they extended the stability of periodic orbits in the hybrid zero dynamics to the full-order dynamics [9]. This method was applied in experiment to establish stable walking of an under-actuated five-link robot subject to impact dynamics at

This material is based upon work supported by the National Science Foundation Graduate Research Fellowship under Grant No. DGE-1745301 and NSF NRI Grant No. 1724464 and by the RoAMS initiative

R. Gehlhar and A. Ames are with the Department of Mechanical and Civil Engineering, California Institute of Technology, Pasadena, CA 91125 USA. (e-mails: $\{$ rgehlhar, ames $\} @$ caltech.edu)

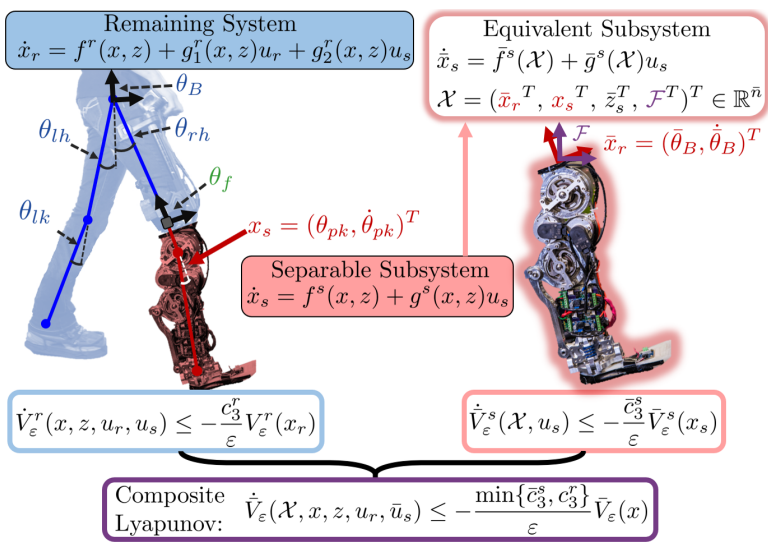

Fig. 1. Human-prosthesis separable system (left) with separable prosthesis subsystem (red) and remaining human system (blue). Equivalent prosthesis subsystem (right) with base coordinates and interaction force inputs. The composite CLF of the remaining system RES-CLF (blue) and equivalent subsystem RES-CLF (red) yields a RES-CLF for the whole system (purple).

foot strike. CLFs also proved useful for robotic walking on hardware when formulated as quadratic programs (QPs) [4], [10]. To apply this powerful nonlinear control technique to powered prostheses, we view the human-prosthesis system as a separable system [8], where a subsystem, namely the prosthesis, is separable from the system since it is not a function of the control input of the remaining system, the human. We examine separating RES-CLFs for separable systems to construct a RES-CLF based on the prosthesis alone with the same stability guarantees established in [9]. Separable Lyapunov functions were termed in [11] to describe stability analysis methods for interconnected nonlinear systems, such as in [12]. Here we construct separable RES-CLFs to define a class of controllers to render provably stable hybrid periodic orbits of nonlinear separable systems with zero dynamics.

The main contributions of this paper are (i) establishing stability guarantees of a hybrid dynamical system with zero dynamics through a subsystem controller relying solely on local information, and (ii) providing a method to construct such controllers. This work enables construction of a class of model-dependent prosthesis controllers, bringing the human in the loop of prosthesis control with strong formal guarantees of stability. Section II provides an overview of hybrid systems, RES-CLFs, and separable systems to establish a composite CLF for a separable system guarantees stability of a hybrid periodic orbit. Section III outlines construction of CLFs for partially feedback linearizable systems that yield a separable form to construct RES-CLFs for separable systems. Section IV describes the amputee-prosthesis model used to demonstrate the results in simulation. 


\section{Hybrid Separable Systems and Zero Dynamics}

In this section we extend exponential stability of a hybrid periodic orbit in the zero dynamics to the full-order dynamics with two RES-CLFs for a separable system, the form of the human-prosthesis system, shown in Fig. 1. We can construct an equivalent prosthesis subsystem, independent of the human, using inputs available from a force sensor and IMU in practice. A RES-CLF for this equivalent subsystem, allows independent construction of the separable subsystem control law relying only on local information and stabilizes the full-order system when the remaining system is known to stabilize itself. To begin, we briefly introduce hybrid systems.

Hybrid Systems. Consider a hybrid control system:

$$
\mathscr{H} \mathscr{C}= \begin{cases}\dot{x}=f(x, z)+g(x, z) u & \text { if }(x, z) \in \mathcal{D} \backslash S \\ \dot{z}=q(x, z) & \\ x^{+}=\Delta_{X}\left(x^{-}, z^{-}\right) & \text {if }\left(x^{-}, z^{-}\right) \in S \\ z^{+}=\Delta_{Z}\left(x^{-}, z^{-}\right) & \end{cases}
$$

where $x \in X \subset \mathbb{R}^{n}$ are controlled (output) states, $z \in Z \subset$ $\mathbb{R}^{n_{z}}$ uncontrolled states, $U \subset \mathbb{R}^{m}$ is a set of admissible control inputs for $u$. The functions $f, g, q, \Delta_{X}, \Delta_{Z}$ are locally Lipschitz in their arguments. The domain $\mathcal{D}$ is a closed subset of $X \times Z$, the guard or switching surface $S \subset \mathcal{D}$ is a co-dimension one submanifold of $\mathcal{D}$, defined by,

$$
\begin{aligned}
& \mathcal{D}=\{(x, z) \in X \times Z: h(x, z) \geq 0\}, \\
& S=\{(x, z) \in X \times Z: h(x, z)=0 \text { and } \dot{h}(x, z)<0\},
\end{aligned}
$$

where the continuously differentiable function $h: X \times Z \rightarrow \mathbb{R}$ yields $L_{g} h=0$. Here we assume $f(0, z)=0$ and $\Delta_{X}(0, z)=$ 0 yielding the surface $Z$ defined by $x=0$ with dynamics $\dot{z}=$ $q(0, z)$ as invariant for the continuous and discrete dynamics. This yields the hybrid system for the hybrid zero dynamics:

$$
\left.\mathscr{H}\right|_{Z}=\left\{\begin{array}{cl}
\dot{z}=q(0, z) & \text { if } z \in Z \backslash(S \cap Z) \\
z^{+}=\Delta_{Z}\left(0, z^{-}\right) & \text {if } z^{-} \in S \cap Z .
\end{array}\right.
$$

RES-CLF. To later evaluate the stability of a periodic orbit for this hybrid system, let us review RES-CLFs, an idea introduced in [9]. A RES-CLF for the continuous dynamics of (1) is a function with positive constants $c_{1}, c_{2}, c_{3}>0$ such that for all $0<\varepsilon<1$ and $(x, z) \in X \times Z$,

$$
\begin{aligned}
& c_{1}\|x\|^{2} \leq V_{\varepsilon}(x) \leq \frac{c_{2}}{\varepsilon^{2}}\|x\|^{2} \\
& \inf _{u \in U}\left[L_{f} V_{\varepsilon}(x, z)+L_{g} V_{\varepsilon}(x, z) u+\frac{c_{3}}{\varepsilon} V_{\varepsilon}(x)\right] \leq 0,
\end{aligned}
$$

where $L_{f} V_{\varepsilon}$ and $L_{g} V_{\varepsilon}$ denote the Lie derivatives [13]. The following set consists of control values that yield $\dot{V}_{\varepsilon}(x, z, u)<$ $-\frac{c_{3}}{\varepsilon} V_{\varepsilon}(x)$, i.e. satisfy (ii):

$K_{\varepsilon}(x, z)=\left\{u \in U: L_{f} V_{\varepsilon}(x, z)+L_{g} V_{\varepsilon}(x, z) u+\frac{c_{3}}{\varepsilon} V_{\varepsilon}(x) \leq 0\right\}$.

With $u^{\varepsilon}(x, z) \in K_{\varepsilon}(x, z)$ for all $x \in X \times Z$ the closed-loop system of the continuous dynamics of (1) becomes:

$$
\mathscr{H}_{\varepsilon}= \begin{cases}\dot{x}=f(x, z)+g(x, z) u^{\varepsilon}(x, z) & \text { if }(x, z) \in \mathcal{D} \backslash S \\ \dot{z}=q(x, z) & \\ x^{+}=\Delta_{X}\left(x^{-}, z^{-}\right) & \text {if }\left(x^{-}, z^{-}\right) \in S . \\ z^{+}=\Delta_{Z}\left(x^{-}, z^{-}\right) & \end{cases}
$$

For the continuous dynamics of (4), let $\varphi_{t}(x, z)$ be its periodic flow and $\mathscr{O}$ its corresponding periodic orbit. For the zero dynamics $\dot{z}=q(0, z)$ with periodic flow $\varphi_{t}^{z}$, let $\mathscr{O}_{Z}$ be its corresponding periodic orbit. Because of the invariance of the zero dynamics surface $Z$ assumption, a periodic orbit for the zero dynamics $\mathscr{O}_{Z}$ corresponds to a periodic orbit for the fullorder dynamics, $\mathscr{O}=\iota_{0}\left(\mathscr{O}_{Z}\right)$, where $\iota_{0}: Z \rightarrow X \times Z$ is the canonical embedding $\iota_{0}(z)=(0, z)$. We saw in [9] how the existence of a RES-CLF guaranteed for an exponentially stable periodic orbit $\mathscr{O}_{Z}$ for the zero dynamics $\left.\mathscr{H}\right|_{Z}$ transverse to $S \cap Z$, the corresponding periodic orbit of the full-order dynamics $\mathscr{O}=\iota_{0}\left(\mathscr{O}_{Z}\right)$ is exponentially stable.

Hybrid Separable Systems. Now let us consider this idea in the context of separable systems by considering the continuous dynamics of (1) to be a separable system as defined in [8] with the addition of zero dynamics:

$$
\begin{gathered}
{\left[\begin{array}{c}
\dot{x}_{r} \\
\dot{x}_{s}
\end{array}\right]=\left[\begin{array}{l}
f^{r}(x, z) \\
f^{s}(x, z)
\end{array}\right]+\left[\begin{array}{cc}
g_{1}^{r}(x, z) & g_{2}^{r}(x, z) \\
0 & g^{s}(x, z)
\end{array}\right]\left[\begin{array}{l}
u_{r} \\
u_{s}
\end{array}\right],} \\
\dot{z}=q(x, z), \\
x_{r} \in \mathbb{R}^{n_{r}}, x_{s} \in \mathbb{R}^{n_{s}}, u_{r} \in \mathbb{R}^{m_{r}}, u_{s} \in \mathbb{R}^{m_{s}},
\end{gathered}
$$

where $n_{r}+n_{s}=n$ and $m_{r}+m_{s}=m$. We define the separable subsystem and remaining system respectively as:

$$
\begin{aligned}
& \dot{x}_{s}=f^{s}(x, z)+g^{s}(x, z) u_{s}, \\
& \dot{x}_{r}=f^{r}(x, z)+g_{1}^{r}(x, z) u_{r}+g_{2}^{r}(x, z) u_{s},
\end{aligned}
$$

where (6) is a function of $u_{s}$, and (7) of both $u_{r}$ and $u_{s}$.

To obtain a RES-CLF for the subsystem that only requires local information, we reintroduce an idea from [8], an equivalent subsystem, this time with zero dynamic coordinates:

$$
\begin{aligned}
& \dot{\bar{x}}_{s}=\bar{f}^{s}(\mathcal{X})+\bar{g}^{s}(\mathcal{X}) u_{s}, \\
& \mathcal{X}=\left(\bar{x}_{r}^{T}, x_{s}^{T}, \bar{z}_{s}^{T}, \mathcal{F}^{T}\right)^{T} \in \mathbb{R}^{\bar{n}} .
\end{aligned}
$$

Here $\bar{x}_{s}=x_{s}, \bar{x}_{r} \in \mathbb{R}^{\bar{n}_{r}}$ are measurable states, and $\mathcal{X}$ is the state vector $\bar{x}=\left(\bar{x}_{r}^{T}, x_{s}^{T}\right)^{T}$ augmented with uncontrollable states $\bar{z}_{s} \in \mathbb{R}^{\bar{n}_{z}}$ and input $\mathcal{F} \in \mathbb{R}^{n_{f}}$. For this subsystem to equate to the separable subsystem (6), we assume there exists a transformation $T: X \times Z \rightarrow \mathbb{R}^{\bar{n}}$ such that $T(x, z)=\mathcal{X}$ and the following conditions hold: $f^{s}(x, z)=\bar{f}^{s}(\mathcal{X})$ and $g^{s}(x, z)=\bar{g}^{s}(\mathcal{X})$. In the next section, we show the existence of individual RES-CLFs for the equivalent subsystem and remaining system yields a RES-CLF for the full-order system implying Theorem 2 of [9] applies to guarantee exponential stability of the hybrid periodic orbit $\mathscr{O}=\iota_{0}\left(\mathscr{O}_{Z}\right)$.

Composite RES-CLF for Separable Systems. Assume there exists a RES-CLF $\bar{V}_{\varepsilon}^{s}\left(x_{s}\right)$ for the equivalent subsystem,

$$
\begin{aligned}
& \bar{c}_{1}^{s}\left\|x_{s}\right\|^{2} \leq \bar{V}_{\varepsilon}^{s}\left(x_{s}\right) \leq \frac{\bar{c}_{2}^{s}}{\varepsilon^{2}}\left\|x_{s}\right\|^{2} \\
& \inf _{u_{s} \in \mathbb{R}^{m_{s}}}\left[L_{\bar{f}^{s}} \bar{V}_{\varepsilon}^{s}(\mathcal{X})+L_{\bar{g}^{s}} \bar{V}_{\varepsilon}^{s}(\mathcal{X}) u_{s}\right] \leq-\frac{\bar{c}_{3}^{s}}{\varepsilon} \bar{V}_{\varepsilon}^{s}\left(x_{s}\right),
\end{aligned}
$$

where $\bar{c}_{1}^{s}, \bar{c}_{2}^{s}$, and $\bar{c}_{3}^{s}$ are positive constants. Here $\bar{V}_{\varepsilon}^{s}\left(x_{s}\right)$ is only a function of the separable subsystem states $x_{s}$ and its derivative $\dot{\bar{V}}_{\varepsilon}^{s}\left(\mathcal{X}, u_{s}\right)$ is based solely on local information. The following set consists of all control values $u_{s}$ that satisfy $\dot{\bar{V}}_{\varepsilon}^{s}\left(\mathcal{X}, u_{s}\right) \leq-\frac{\bar{c}_{3}^{s}}{\varepsilon} \bar{V}_{\varepsilon}^{s}\left(x_{s}\right)$ :

$\bar{K}_{\varepsilon}^{s}(\mathcal{X})=\left\{u_{s} \in \mathbb{R}^{m_{s}}: L_{\bar{f}^{s}} \bar{V}_{\varepsilon}^{s}(\mathcal{X})+L_{\bar{g}^{s}} \bar{V}_{\varepsilon}^{s}(\mathcal{X}) u_{s} \leq-\frac{\bar{c}_{s}^{s}}{\varepsilon} \bar{V}_{\varepsilon}^{s}\left(x_{s}\right)\right\}$. 
Let us also assume there exists a RES-CLF $V_{\varepsilon}^{r}\left(x_{r}\right)$ for the remaining system such that, given a $u_{s} \in \bar{K}_{\varepsilon}^{s}(\mathcal{X})$,

$$
\begin{aligned}
c_{1}^{r}\left\|x_{r}\right\|^{2} \leq V_{\varepsilon}^{r}\left(x_{r}\right) \leq & \frac{c_{2}^{r}}{\varepsilon^{2}}\left\|x_{r}\right\|^{2} \\
\inf _{u_{r} \in \mathbb{R}^{m_{r}}}\left[L_{f^{r}} V_{\varepsilon}^{r}(x, z)\right. & +L_{g_{1}^{r}} V_{\varepsilon}^{r}(x, z) u_{r} \\
& \left.+L_{g_{2}^{r}} V_{\varepsilon}^{r}(x, z) u_{s}\right] \leq-\frac{c_{3}^{r}}{\varepsilon} V_{\varepsilon}^{r}\left(x_{r}\right),
\end{aligned}
$$

where $c_{1}^{r}, c_{2}^{r}$, and $c_{3}^{r}$ are positive constants. Here $V_{\varepsilon}^{r}\left(x_{r}\right)$ is only a function of the remaining states $x_{r}$ and its derivative $\dot{V}_{\varepsilon}^{r}(x, z, u)$ depends on both control inputs $\left(u_{r}, u_{s}\right)$. The following set consists of all control values $u_{r}$ that satisfy $\dot{V}_{\varepsilon}^{r}(x, z, u) \leq-\frac{c_{3}^{r}}{\varepsilon} V_{\varepsilon}^{r}\left(x_{r}\right)$ for $u_{s} \in \bar{K}_{\varepsilon}^{s}(\mathcal{X})$ :

$$
\begin{aligned}
K_{\varepsilon}^{r}(x, z)=\left\{u_{r} \in \mathbb{R}^{m_{r}}\right. & : L_{f^{r}} V_{\varepsilon}^{r}(x, z)+L_{g_{1}^{r}} V_{\varepsilon}^{r}(x, z) u_{r} \\
& \left.+L_{g_{2}^{r}} V_{\varepsilon}^{r}(x, z) u_{s} \leq-\frac{c_{3}^{r}}{\varepsilon} V_{\varepsilon}^{r}\left(x_{r}\right)\right\}\left.\right|_{u_{s} \in \bar{K}_{\varepsilon}^{s}(\mathcal{X})} .
\end{aligned}
$$

Theorem 1: Let $\mathscr{O}_{Z}$ be an exponentially stable periodic orbit of the hybrid zero dynamics $\left.\mathscr{H}\right|_{Z}$ transverse to $S \cap Z$ and assume there exists RES-CLFs $\bar{V}_{\varepsilon}^{s}\left(x_{s}\right)$ and $V_{\varepsilon}^{r}\left(x_{r}\right)$ for the equivalent subsystem (8) and remaining system (7), respectively, of the continuous dynamics of $\mathscr{H} \mathscr{C}$ (1). Then there exists an $\bar{\varepsilon}>0$ such that for all $0<\varepsilon<\bar{\varepsilon}$ and for all Lipschitz continuous $u_{s}^{\varepsilon}(\mathcal{X}) \in \bar{K}_{\varepsilon}^{s}(\mathcal{X})$ and respective $\left.u_{r}^{\varepsilon}(x, z) \in K_{\varepsilon}^{r}(x, z)\right|_{u_{s} \in \bar{K}_{\varepsilon}^{s}(\mathcal{X})}, \mathscr{O}=\iota_{0}\left(\mathscr{O}_{Z}\right)$ is an exponentially stable hybrid periodic orbit of $\mathscr{H}_{\varepsilon}$ with $u^{\varepsilon}(\mathcal{X}, x, z)=$ $\left(u_{r}^{\varepsilon T}(x, z), u_{s}^{\varepsilon T}(\mathcal{X})\right)^{T}$.

Proof: We show the conditions listed above are within the conditions of Theorem 2 of [9], so the same result holds. First we show that given a RES-CLF for the equivalent subsystem and remaining system, there exists a RES-CLF for the whole system. Consider the composite Lyapunov function:

$$
\bar{V}_{\varepsilon}(x)=\bar{V}_{\varepsilon}^{s}\left(x_{s}\right)+V_{\varepsilon}^{r}\left(x_{r}\right) \leq \frac{\max \left\{\bar{c}_{2}^{s}, c_{2}^{r}\right\}}{\varepsilon^{2}}\|x\|^{2},
$$

Similarly $\bar{V}_{\varepsilon}(x) \geq \min \left\{\bar{c}_{1}^{s}, c_{1}^{r}\right\}\|x\|^{2}$, satisfying (i). For (ii),

$$
\begin{aligned}
\dot{\bar{V}}_{\varepsilon}\left(\mathcal{X}, x, z, u_{r}, u_{s}\right) & =\dot{\bar{V}}_{\varepsilon}^{s}\left(\mathcal{X}, u_{s}\right)+\dot{V}_{\varepsilon}^{r}\left(x, z, u_{r}\right) \\
& \leq-\frac{\min \left\{\bar{c}_{3}^{s}, c_{3}^{r}\right\}}{\varepsilon} \bar{V}_{\varepsilon}(x)
\end{aligned}
$$

establishing $\bar{V}_{\varepsilon}(x)$ as a RES-CLF of the continuous dynamics of (1). Dropping the arguments $\left(\mathcal{X}, u_{s}\right)$ and $\left(x, z, u_{r}\right)$ for simplicity's sake, we next show for $\bar{u}^{\varepsilon}=\left(u_{r}^{\varepsilon T}, u_{s}^{\varepsilon T}\right)^{T}$, where $u_{r}^{\varepsilon} \in K_{\varepsilon_{r}}^{r}$ and $u_{s}^{\varepsilon} \in \bar{K}_{\varepsilon}^{s}$, that $\bar{u}^{\varepsilon} \in K_{\varepsilon}$ by ensuring $\dot{\bar{V}}_{\varepsilon} \leq-\frac{\min \left\{\bar{c}_{3}^{s}, c_{3}^{r}\right\}}{\varepsilon} \bar{V}_{\varepsilon}(x)$. Using this $u_{r}^{\varepsilon}$ and $u_{s}^{\varepsilon}$ and building on what was shown above,

$$
\begin{aligned}
\dot{\bar{V}}_{\varepsilon} & =\dot{\bar{V}}_{\varepsilon}^{s}+\dot{V}_{\varepsilon}^{r} \\
& =\left(L_{\bar{f}^{s}} \bar{V}_{\varepsilon}^{s}+L_{\bar{g}^{s}} \bar{V}_{\varepsilon}^{s} u_{s}^{\varepsilon}\right)+\left(L_{f^{r}} V_{\varepsilon}^{r}+L_{g_{1}^{r}} V_{\varepsilon}^{r} u_{r}^{\varepsilon}+L_{g_{2}^{r}} V_{\varepsilon}^{r} u_{s}^{\varepsilon}\right) \\
& \leq\left(-\frac{\bar{c}_{3}^{s}}{\varepsilon} \bar{V}_{\varepsilon}^{s}\left(x_{s}\right)\right)+\left(-\frac{c_{3}^{r}}{\varepsilon} V_{\varepsilon}^{r}\left(x_{r}\right)\right) \\
& \leq-\frac{\min \left\{\bar{c}_{3}^{s}, c_{3}^{r}\right\}}{\varepsilon} \bar{V}_{\varepsilon}(x),
\end{aligned}
$$

hence $\bar{u}^{\varepsilon}=\left(u_{r}^{\varepsilon T}, u_{s}^{\varepsilon T}\right)^{T} \in K_{\varepsilon}(x, z)$. Since these conditions fit within the conditions of Theorem 2 of [9], the same result applies: $\mathscr{O}=\iota_{0}\left(\mathscr{O}_{Z}\right)$ is exponentially stable for $\mathscr{H}_{\varepsilon}$.
Remark. In Section IV, we prescribe limit cycle motion matching human data to the human model with a RESCLF controller. Research on central pattern generators suggest biological walkers such as humans exhibit stable rhythmic behavior, meaning they have limit cycles [14]. Thus we do not make biomechanical claims of the human's control method, but instead prescribe a stable limit cycle to approximate human walking. Our class of RES-CLF controllers encompasses all controllers that stabilize these hybrid limit cycles; for control purposes we find it reasonable to assume the human's effective control input is within our class of control laws for the remaining system. Then by Theorem 1, a RES-CLF prosthesis subsystem controller with only local information will guarantee the entire system is stable.

\section{SeParable CLF CONSTRUCtion}

To obtain RES-CLFs for separable systems, we begin with constructing CLFs for partially feedback linearizable systems, an idea introduced in [13, pp. 160-172]. Our construction yields a separable form that allows us to independently stabilize each output while guaranteeing full-order system stability. This method also provides a basis to construct separable RESCLFs for separable systems.

Output Dynamics and Feedback Linearization. Any partially feedback linearizable system can be converted to normal form per methods introduced in [6, pp. 407-411]. We assume our system (1) is in normal form for the linearly independent outputs $y: \mathbb{R}^{n} \rightarrow \mathbb{R}^{k}$, where $k=m$ to obtain a square system [6, p. 407]. These outputs are of vector relative degree $\vec{\gamma}=\left(\gamma_{1}, \gamma_{2}, \ldots, \gamma_{k}\right)$, and yield output dynamics of the dimension of our system states, i.e. $\sum_{i=1}^{k} \gamma_{i}=n$. We write the output dynamics as the continuous dynamics of (1):

$$
\left\{\begin{array} { r l } 
{ x _ { i } ^ { 1 } } & { = y _ { i } ( x , z ) } \\
{ x _ { i } ^ { j + 1 } } & { = \dot { x } _ { i } ^ { j } } \\
{ \dot { x } _ { i } ^ { \gamma _ { i } } } & { = a _ { i } ( x , z ) + b _ { i } ( x , z ) u } \\
{ \forall i } & { \in \{ 1 \cdots m \} , j \in \{ 1 \cdots \gamma _ { i } - 1 \} }
\end{array} \Rightarrow \left\{\begin{array}{l}
\dot{x}^{\vec{\gamma}}=\left(x_{1}^{\gamma_{1} T}, x_{2}^{\gamma_{2} T}, \cdots, x_{k}^{\gamma_{k} T}\right)^{T} \\
\dot{x}^{\vec{\gamma}}=\hat{a}(x, z)+\hat{b}(x, z) u,
\end{array}\right.\right.
$$

from which we construct the feedback linearizing controller:

$$
u=-\hat{b}^{-1}(x, z)(\hat{a}(x, z)-\mu),
$$

with the auxiliary control input $\mu$. Here $\hat{b}^{-1}(x, z)$ is invertible because the outputs are linearly independent and the system is square. Applying this controller results in $\dot{x} \vec{\gamma}=\mu$ and we rewrite our output dynamics:

$$
\begin{aligned}
\dot{x}_{i} & =\underbrace{\left[\begin{array}{cc}
0 & I_{\gamma_{i}-1 \times \gamma_{i}-1} \\
0 & 0
\end{array}\right]}_{F_{i}} x_{i}+\underbrace{\left[\begin{array}{c}
0_{\gamma_{i}-1 \times 1} \\
1
\end{array}\right]}_{G_{i}} \mu_{i} \\
\dot{x} & =F x+G \mu,
\end{aligned}
$$

where $x=\left(x_{1}^{T}, x_{2}^{T}, \ldots, x_{k}^{T}\right)^{T}, \mu=\left(\mu_{1}^{T}, \mu_{2}^{T}, \ldots, \mu_{k}^{T}\right)^{T}$, and

$$
\begin{aligned}
& F=\operatorname{diag}\left(F_{1}, \ldots, F_{k}\right) \in \mathbb{R}^{n \times n}, \\
& G=\operatorname{diag}\left(G_{1}, \ldots, G_{k}\right) \in \mathbb{R}^{n \times k},
\end{aligned}
$$

where $\operatorname{diag}()$ notates a block diagonal matrix of listed elements. This $F$ and $G$ yields a full rank controllability matrix 
enabling CLF construction with the continuous-time Algebraic Riccati equation (CARE).

Separable CLFs. To enable independent controller construction for the human and prosthesis subsystems, we construct a CLF we can separate for each output $x_{i}$, starting with a lemma on the structure of the CARE solution.

Lemma 1: For any $F$ and $G$ of the form (14) and weight matrix $Q=\operatorname{diag}\left(Q_{1}, \ldots, Q_{k}\right)$, where $Q_{i} \in \mathbb{R}^{\gamma_{i} \times \gamma_{i}}$, a solution to the CARE equation,

$$
F^{T} P+P F+P G G^{T} P+Q=0,
$$

is a block diagonal positive definite matrix $P=$ $\operatorname{diag}\left(P_{1}, \ldots, P_{k}\right)$ with elements $\left\{P_{i} \in \mathbb{R}^{\gamma_{i} \times \gamma_{i}}\right\}_{i=1, \ldots, k}$.

Proof: Let us assume $P$ is of the given form, this would yield the left side of (15) to be a block diagonal matrix of the following set of components:

$$
\left\{F_{i}^{T} P_{i}+P_{i} F_{i}+P_{i} G_{i} G_{i}^{T} P_{i}+Q_{i}\right\}_{i=1 \ldots k},
$$

where the right side of (15) equates each of these components to 0 . This takes the form of CARE and since $F_{i}$ and $G_{i}$ of the form (14) yield a full rank controllability matrix, there exists a solution $P_{i}$ for $i=1 \ldots k$. Hence this block diagonal structure of $P$ satisfies (15) and is therefore a solution.

We know from [9] that for a $P$ from CARE, $V(x)=x^{T} P x$ is a CLF satisfying

$$
\inf _{\mu}\left[L_{F} V(x)+L_{G} V(x) \mu\right] \leq-\frac{\lambda_{\min }(Q)}{\lambda_{\max }(P)} V(x),
$$

where $L_{F} V(x)=x\left(F^{T} P+P F\right) x$ and $L_{G} V(x)=2 x^{T} P G$. Based on this construction, we can separate our CLF such that for each output $x_{i}$ we can define a CLF $V^{i}\left(x_{i}\right)$, or sub-CLF, with sub-components from our separable CLF $V(x)$.

Definition 1: A separable CLF is a CLF $V_{\mathrm{sep}}(x)=x^{T} P x$ with $P$ of the form in Lemma 1, satisfying (15) for $F$ and $G$ of (14) and $Q$ of the form in Lemma 1, where $k \geq 2$.

Definition 2: A sub-CLF is a function $V_{\mathrm{sub}}\left(x_{\mathrm{sub}}\right)$ :

$$
\begin{gathered}
V_{\mathrm{sub}}\left(x_{\mathrm{sub}}\right):=V^{i}\left(x_{i}\right)=x_{i}^{T} P_{i} x_{i} \\
\inf _{\mu \in \mathbb{R}}\left[L_{F} V^{i}\left(x_{i}\right)+L_{G} V^{i}\left(x_{i}\right) \mu_{i}\right] \leq-\frac{\lambda_{\min }\left(Q_{i}\right)}{\lambda_{\max }\left(P_{i}\right)} V^{i}\left(x_{i}\right),
\end{gathered}
$$

where $L_{F} V^{i}\left(x_{i}\right)=x_{i}\left(F_{i}^{T} P_{i}+P_{i} F_{i}\right) x$ and $L_{G} V^{i}\left(x_{i}\right)=$ $2 x^{T} P_{i} G_{i}$. for $i \in\{1 \cdots k\}$ for a separable CLF $V_{\mathrm{sep}}(x)$.

Based on these definitions, we now establish constructing the auxiliary control inputs $\mu_{i}$ to satisfy their sub-CLF conditions will yield a $\mu$ satisfying the separable CLF condition.

Theorem 2: Given a separable $C L F V_{\mathrm{sep}}(x)$, if for all $i \in$ $\{1 \ldots k\}, \mu_{i}$ satisfies its sub-CLF condition (17), then $\mu=$ $\left[\mu_{1}^{T}, \mu_{2}^{T}, \cdots, \mu_{k}^{T}\right]^{T}$ satisfies (16) for $V_{\text {sep }}(x)$.

Proof: Since $P$ in $V_{\text {sep }}(x)$ is the specified block diagonal structure in Lemma 1 and satisfies (15), each $P_{i}$ must satisfy:

$$
F_{i}^{T} P_{i}+P_{i} F_{i}+P_{i} G_{i} G_{i}^{T} P_{i}+Q_{i}=0,
$$

and hence forms a CLF satisfying the sub-CLF conditions (17). We write $V_{\text {sep }}(x)$ as a composite CLF of sub-CLFs:

$V_{\text {sep }}(x)=x_{1}^{T} P_{1} x_{1}+x_{2}^{T} P_{2} x_{2}+\cdots+x_{k}^{T} P_{k} x_{k}=\sum_{i=1}^{k} V^{i}\left(x_{i}\right)$,

where $V^{i}\left(x_{i}\right)=x_{i}^{T} P_{i} x_{i}$. Taking the infimum of the derivative, we bound the separable CLF in terms of the sub-CLFs:

$$
\begin{aligned}
\inf _{\mu \in \mathbb{R}^{k}}\left[L_{F} V_{\text {sep }}(x)+L_{G} V_{\text {sep }}(x) \mu\right] & =\sum_{i=1}^{k} \inf \left[L_{F} V^{i}\left(x_{i}\right)+L_{G} V^{i}\left(x_{i}\right) \mu_{i}\right] \\
& \leq \sum_{i=1}^{k}-\frac{\lambda_{\min }\left(Q_{i}\right)}{\lambda_{\max }\left(P_{i}\right)} V^{i}\left(x_{i}\right) .
\end{aligned}
$$

Comparing the elements in this bound to the separable CLF bound in (16), we note $V_{\text {sep }}(x)=\sum_{i=1}^{k} V^{i}\left(x_{i}\right), \lambda_{\min }\left(Q_{i}\right) \geq$ $\lambda_{\min }(Q)$, and $\lambda_{\max }\left(P_{i}\right) \leq \lambda_{\max }(P)$ for all $i=1, \ldots, k$. Hence:

$$
\sum_{i=1}^{k}-\frac{\lambda_{\min }\left(Q_{i}\right)}{\lambda_{\max }\left(P_{i}\right)} V^{i}\left(x_{i}\right) \leq-\frac{\lambda_{\min }(Q)}{\lambda_{\max }(P)} V_{\mathrm{sep}}(x) .
$$

Therefore any set $\left\{\mu_{i} \in \mathbb{R}\right\}_{i=1, \ldots, k}$ that satisfies each respective sub-CLF condition (17), will also satisfy the CLF condition (16) for the separable CLF.

This CLF construction allows us to develop $\mu_{i}$ with only knowledge of $x_{i}$ to stabilize each output while guaranteeing stability of the whole system. For the human-prosthesis system, we can construct these inputs separately for the human and prosthesis. To apply Theorem 1, we now extend this method to develop RES-CLFs for separable systems.

Separable RES-CLFs for Separable Systems. For a system with $k$ relative degree 2 outputs, common in mechanical systems, we can transform each sub-CLF to a sub-RES-CLF following the method in [9] with $0<\varepsilon<1$ :

$$
V_{\varepsilon}^{i}(x)=x_{i}^{T}\left[\begin{array}{cc}
\frac{1}{\varepsilon} I & 0 \\
0 & I
\end{array}\right] P_{i}\left[\begin{array}{cc}
\frac{1}{\varepsilon} I & 0 \\
0 & I
\end{array}\right] x_{i}=: x_{i}^{T} P_{i}^{\varepsilon} x_{i} .
$$

By Theorem 1's proof constructions, we conclude the summation of sub-RES-CLFs yields a RES-CLF for the whole system. Repeating Theorem 2's proof would establish the same result for this separable RES-CLF. By summing the sub-RESCLFs for the separable subsystem outputs we attain a RESCLF $\bar{V}_{\varepsilon}^{s}\left(x_{s}\right)=x_{s}^{T} P_{s}^{\varepsilon} x_{s}$ for the equivalent subsystem (8) and with the remaining outputs a RES-CLF $V_{\varepsilon}^{r}\left(x_{r}\right)=x_{r}^{T} P_{r}^{\varepsilon} x_{r}$ for the remaining system (7), where $P_{r}^{\varepsilon}$ and $P_{s}^{\varepsilon}$ are diagonal matrices of elements $P_{i}^{\varepsilon}$ for their respective outputs.

To find a subsystem control law in $\bar{K}_{\varepsilon}^{s}(\mathcal{X})$, we need the subsystem output dynamics. Due to a separable system's structure (5), we know $\dot{x}_{s}^{\gamma^{s}}$ is independent of $u_{s}$ yielding (11) of the form,

$$
\left[\begin{array}{c}
\dot{x}_{\vec{\gamma}}^{\vec{\gamma}^{r}} \\
\dot{x}_{s}^{\vec{\gamma}^{s}}
\end{array}\right]=\left[\begin{array}{l}
\hat{a}^{r}(x, z) \\
\hat{a}^{s}(x, z)
\end{array}\right]+\left[\begin{array}{cc}
\hat{b}_{1}^{r}(x, z) & \hat{b}_{2}^{r}(x, z) \\
0 & \hat{b}^{s}(x, z)
\end{array}\right]\left[\begin{array}{l}
u_{r} \\
u_{s}
\end{array}\right],
$$

where $\vec{\gamma}^{s}$ and $\vec{\gamma}^{r}$ are the vector relative degrees of the separable subsystem and remaining system outputs, respectively, as defined in [8]. As stated in [8], having an equivalent subsystem enables construction of feedback linearizing terms, 
$\hat{\bar{a}}^{s}(\mathcal{X})$ and $\hat{\bar{b}}^{s}(\mathcal{X})$, of separable outputs in terms of the equivalent subsystem, such that for all $x$ and $z$,

$$
\hat{\bar{a}}^{s}(\mathcal{X})=\hat{a}^{s}(x, z) \quad \text { and } \quad \hat{\bar{b}}^{s}(\mathcal{X})=\hat{b}^{s}(x, z) .
$$

Using these terms, we formulate a QP for the subsystem control law $u_{s}$ without knowledge of the rest of the system:

$$
\begin{array}{r}
u_{s}^{\star}=\underset{u_{s} \in \mathbb{R}^{m_{s}}}{\operatorname{argmin}} u_{s}^{T} \hat{\bar{b}}^{s}(\mathcal{X})^{T} \hat{\bar{b}}^{s}(\mathcal{X}) u_{s}+\hat{\bar{a}}^{s}(\mathcal{X}) u_{s} \\
\text { s.t. } L_{F_{s}} \bar{V}_{\varepsilon}^{s}\left(x_{s}\right)+L_{G_{s}} \bar{V}_{\varepsilon}^{s}\left(x_{s}\right)\left(\hat{\bar{b}}^{s}(\mathcal{X}) u_{s}\right. \\
\left.\quad+\hat{\bar{a}}^{s}(\mathcal{X})\right) \leq-\frac{\lambda_{\min }\left(Q_{s}\right)}{\varepsilon \lambda_{\max }\left(P_{s}\right)} \bar{V}_{\varepsilon}^{s}\left(x_{s}\right),
\end{array}
$$

where again $F_{s}, G_{s}, Q_{s}$, and $P_{s}$ are diagonal matrices of the elements $F_{i}, G_{i}, Q_{i}$, and $P_{i}$, respectively, for the subsystem outputs. These constructions work for any separable system with separable outputs, since its normal form is also separable. Inherently $F$ and $G$ of (14) comply with separable form and the feedback linearizing terms of (11) are separable, as shown in the proof of Theorem 1 in [8].

This subsystem control law relies solely on local information and guarantees stability of a hybrid periodic orbit of the zero dynamics in the full-order dynamics when the remaining system is stable. This QP formulation allows torque bounds and model-based constraints to be added, as in [4], [10].

\section{Amputee-Prosthesis Application}

In this section we apply the controller (20) to a planar amputee-prosthesis model to demonstrate the results of the theorems. The model is comprised of 6 links: torso, 2 human thighs and calf, and prosthesis calf and partial thigh. The connection between the prosthesis partial thigh and human right thigh is modeled as a 3 DOF fixed joint $(x, z$ Cartesian position and pitch). This model yields a separable system. Any open-chain robotic system can be modeled as such by dividing the original model into two subsystems and constraining them to each other through a holonomic constraint. The human parameters are estimated with a subject's height and weight and the parameters in [15], [16], for details see [8]. The prosthesis parameters are based on a powered transfemoral prosthesis platform AMPRO3 [17]. We consider 4 actuators: 3 at the human's hips and knee that constitute $u_{r}$, and 1 at the prosthesis knee that constitutes $u_{s}$. The torso is not actuated, introducing zero dynamics into the system. The feet are modeled as point contacts. In practice, we can emulate a point foot model on a transfemoral prosthesis by treating the ankle as a passive spring-damper.

Human-Prosthesis Dynamics. The generalized coordinates for the system are $\theta=\left(\theta_{B}, \theta_{h}, \theta_{f}, \theta_{p k}\right)$, where the extended coordinates $\theta_{B} \in S E(2)$ represent the position and rotation of the robot's base frame; $\theta_{h}=\left(\theta_{l h}, \theta_{l k}, \theta_{r h}\right)$ are the human's left hip, left knee, and right hip, respectively; $\theta_{f}$ are the fixed joint coordinates; and $\theta_{p k}$ is the prosthesis knee. See Fig. 1. With these coordinates, the full-order system constrained dynamics are given by the Euler-Lagrange equation [18],

$$
\begin{aligned}
& D(\theta) \ddot{\theta}+H(\theta, \dot{\theta})=B u+J^{T}(\theta) F \\
& \dot{J}(\theta, \dot{\theta}) \dot{\theta}+J(\theta) \ddot{\theta}=0,
\end{aligned}
$$

where $J(\theta)$ is the Jacobian of the holonomic constraints modeling the foot contact and the fixed joints. We focus on the prosthesis as our separable subsystem and rearrange the dynamics per the methods of [8] to be a separable system (5), where $x_{r}=\left(\theta_{B}, \theta_{h}, \theta_{f}, \dot{\theta}_{B}, \dot{\theta}_{h}, \dot{\theta}_{f}\right)$ and $x_{s}=\left(\theta_{p k}, \dot{\theta}_{p k}\right)$.

Prosthesis Subsystem Dynamics. The generalized coordinates for the prosthesis are $\bar{\theta}=\left(\bar{\theta}_{B}, \theta_{p k}\right)$. Here $\bar{\theta}_{B} \in S E(2)$ are the base coordinates of the prosthesis located at its attachment point. Its constrained dynamics are

$$
\begin{aligned}
& \bar{D}(\bar{\theta}) \ddot{\bar{\theta}}+\bar{H}(\bar{\theta}, \dot{\bar{\theta}})=\bar{B} u_{s}+\bar{J}^{T}(\bar{\theta}) \bar{F}(\bar{\theta}, \dot{\bar{\theta}})+\bar{J}_{f}^{T}(\bar{\theta}) F_{f}, \\
& \dot{\bar{J}}(\bar{\theta}, \dot{\bar{\theta}}) \dot{\bar{\theta}}+\bar{J}(\bar{\theta}) \ddot{\bar{\theta}}=0,
\end{aligned}
$$

where $\bar{J}(\bar{\theta})$ is the Jacobian of the holonomic constraint for the foot contact and $\bar{J}_{f}$ is the projection of the measured socket force $F_{f}$ onto the attachment point. We use the transformation $T(x, z)=\mathcal{X}$ from [8] and rearrange the dynamics as in [8] to be an equivalent subsystem (8). Here the measurable states $\bar{x}_{r}=\left(\bar{\theta}_{B}^{T}, \dot{\bar{\theta}}_{B}^{T}\right)^{T}$ can be obtained with an IMU and the input $\mathcal{F}=F_{f}$ with a force sensor, see Fig. 1. There are no uncontrollable states $\bar{z}_{s}$ for the prosthesis.

Output Functions. Because of the asymmetrical humanprosthesis system, we use two domains to model the steps, with subscript $v$ denoting a domain and $e$ the guard between domains. For the guards (3), $h_{v}(x, z)$ is the height of the nonstance foot. For details on multi-domain hybrid systems see [19]. To construct the separable RES-CLF of Section III, we design relative degree 2 separable outputs [8],

$$
y_{v}\left(x, \alpha_{v}\right)=\left[\begin{array}{c}
y_{v}^{r}\left(x, \alpha_{v}\right) \\
y_{v}^{s}\left(x_{s}, \alpha_{v}\right)
\end{array}\right]=\left[\begin{array}{c}
y^{a, r}(x)-y_{v}^{d, r}\left(x, \alpha_{v}\right) \\
y^{a, s}\left(x_{s}\right)-y_{v}^{d, s}\left(x_{s}, \alpha_{v}\right)
\end{array}\right],
$$

such that the $k_{s}$ number of subsystem outputs is the prosthesis knee angle, $y^{a, s}\left(x_{s}\right)=\theta_{p k}$, and the $k_{r}$ number of remaining outputs are the human's hips and knees, $y^{a, r}(x)=$ $\left(\theta_{l h}, \theta_{l k}, \theta_{r h}\right)^{T}$. For the desired outputs $y_{v}^{d, r}$ and $y_{v}^{d, s}, \alpha_{v}$ are coefficients for Bézier polynomials chosen to match human walking data [20] and such that the zero dynamics surface $Z$ is invariant and contains an exponentially stable periodic orbit $\mathscr{O}_{Z}$ transverse to the switching surface $S \cap Z$, satisfying the assumption of Theorem 1.

Results. To encode the human-like walking trajectories in the human simulation, we use the feedback linearizing controller (12) where $\mu=\left(\mu_{r}^{T}, \mu_{s}^{T}\right)^{T}$, with $\mu_{s}$ as the min-norm controller satisfying the RES-CLF condition for $\bar{V}_{\varepsilon}^{s}\left(x_{s}\right)$, and

$$
\mu_{r}=-\frac{1}{\varepsilon^{2}} y_{v}^{r}(x)-\frac{1}{\varepsilon} \dot{y}_{v}^{r}(x),
$$

which indeed yields a RES-CLF as shown in [9] for this remaining system. The prosthesis tracks its trajectory with the subsystem controller (20), which yields the same $\mu_{s}$ used for the remaining system controller. Simulating this system for 20 steps starting at a perturbed initial condition the prosthesis (subsystem) states settle into a stable periodic orbit, shown in Fig. 2a, demonstrating the rapid convergence of this controller. This figure also depicts the stable periodic orbit of the zero dynamics, demonstrating the exact result of Theorem 1: a stable hybrid periodic orbit of the zero dynamics is guaranteed exponentially stable in the full-order dynamics for 

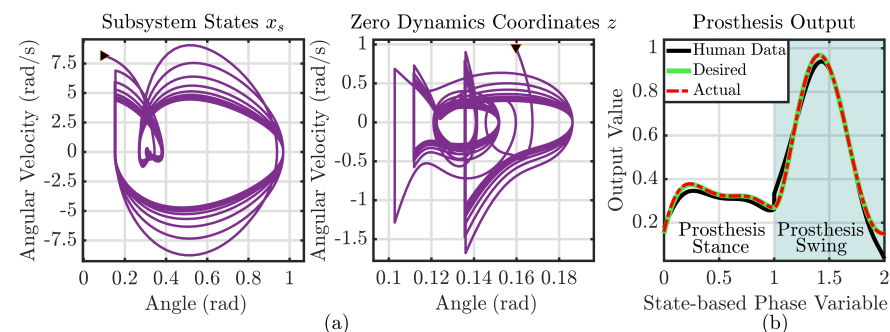

(a)

Fig. 2. (a) Phase portraits of subsystem states (left) and zero dynamics coordinates (right) showing stability for 20 steps with perturbed initial condition (triangle). (b) Actual output prosthesis trajectory $y_{v}^{a, s}$ tracking desired trajectory $y_{v}^{d, s}$, designed to match human data.
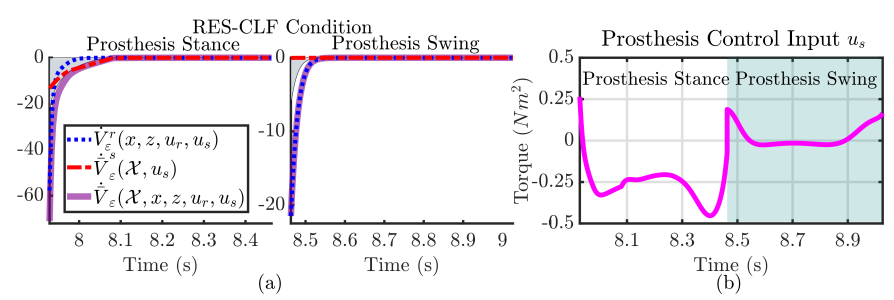

Fig. 3. (a) RES-CLF derivatives for remaining system (blue) and subsystem (red) show convergence for prosthesis stance domain (left) and prosthesis swing (right) and yield a RES-CLF for the full-order system (purple) satisfying its RES-CLF bound (gray). (b) Prosthesis control input from CLF-QP.

controllers of their respective RES-CLF controller classes. Fig. $2 \mathrm{~b}$ shows the output tracking of the prosthesis controller and its relation to human knee data with respect to a state-based parameterization of time [8]. Fig. 3a depicts the RES-CLF derivatives for the subsystems and full-order system, with the full-order system's bound. This demonstrates Theorem 2 by showing the auxiliary control inputs for the remaining system and separable subsystem that satisfy their respective sub-CLF conditions also satisfy the CLF condition for the full-order system. Fig. 3b shows the prosthesis control input from (20) is smooth for each domain and remains in a reasonable range. Fig. 4 shows gait tiles of the human-prosthesis system walking in simulation.

\section{CONCLUSION}

This paper extended RES-CLFs to separable systems to establish exponential stability of a hybrid periodic orbit of the zero dynamics in the full-order dynamics with a subsystem controller constructed solely with local information. Following, we developed a method to construct such RESCLFs for separable systems. This method also outlined constructing CLFs for linearized systems to stabilize each output independent of the rest of the system, while guaranteeing stability of the whole system. This work is significant since it enables construction of a class of model-dependent prosthesis controllers using only locally available sensor information. With the assumption the human can stabilize itself, these controllers provide formal guarantees of exponential stability for a hybrid human-prosthesis system with zero dynamics. This class of controllers gives freedom to design controllers with fast convergence in the presence of disturbances and robustness to force sensor noise to be physically realizable. An example is the robust CLF-QP in [20] which uses an estimate of the range of interaction forces between the human and

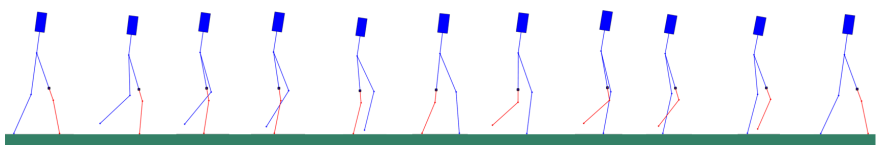

Fig. 4. Gait tiles of human-prosthesis system, prosthesis in red, demonstrating human-like walking in simulation.

prosthesis in place of a force sensor. This controller guarantees stability of the prosthesis for any force within the range. Future work includes implementing this class of controllers on a prosthesis platform.

\section{REFERENCES}

[1] F. Sup, A. Bohara, and M. Goldfarb, "Design and control of a powered transfemoral prosthesis," The International Journal of Robotics Research, vol. 27, no. 2, pp. 263-273, 2008, pMID: 19898683.

[2] H. Zhao, J. Horn, J. Reher, V. Paredes, and A. D. Ames, "First steps toward translating robotic walking to prostheses: a nonlinear optimization based control approach," Autonomous Robots, vol. 41, no. 3, pp. 725-742, 2017.

[3] R. D. Gregg, T. Lenzi, L. J. Hargrove, and J. W. Sensinger, "Virtual constraint control of a powered prosthetic leg: From simulation to experiments with transfemoral amputees," IEEE Transactions on Robotics, vol. 30, no. 6, pp. 1455-1471, Dec 2014.

[4] J. Reher, C. Kann, and A. D. Ames, "An inverse dynamics approach to control lyapunov functions," arXiv preprint arXiv:1910.10824v1, 2019.

[5] H. Khalil, Nonlinear Systems, ser. Pearson Education. Prentice Hall, 2002.

[6] S. Sastry, Nonlinear Systems: Analysis, Stability, and Control, ser. Interdisciplinary Applied Mathematics. Springer New York, 2013.

[7] A. E. Martin and R. D. Gregg, "Hybrid invariance and stability of a feedback linearizing controller for powered prostheses," in 2015 American Control Conference (ACC), July 2015, pp. 4670-4676.

[8] R. Gehlhar, J. Reher, and A. D. Ames, "Control of separable subsystems with application to prostheses," arXiv preprint arXiv:1909.03102v1, 2019.

[9] A. D. Ames, K. Galloway, K. Sreenath, and J. W. Grizzle, "Rapidly exponentially stabilizing control Lyapunov functions and hybrid zero dynamics," IEEE Transactions on Automatic Control, vol. 59, no. 4, pp. 876-891, 2014.

[10] K. Galloway, K. Sreenath, A. D. Ames, and J. W. Grizzle, "Torque saturation in bipedal robotic walking through control lyapunov functionbased quadratic programs," IEEE Access, vol. 3, pp. 323-332, 2015.

[11] A. Rantzer, B. S. Rüffer, and G. Dirr, "Separable lyapunov functions for monotone systems," in 52nd IEEE Conference on Decision and Control, Dec 2013, pp. 4590-4594.

[12] H. Ito, Z. Jiang, S. N. Dashkovskiy, and B. S. Rüffer, "Robust stability of networks of iiss systems: Construction of sum-type lyapunov functions," IEEE Transactions on Automatic Control, vol. 58, no. 5, pp. 1192-1207, May 2013.

[13] A. Isidori, Nonlinear Control Systems, 3rd ed. Berlin, Heidelberg: Springer-Verlag, 1995.

[14] G. Taga, "A model of the neuro-musculo-skeletal system for human locomotion,” Biol. Cybern., vol. 73, no. 2, p. 113-121, Jul. 1995.

[15] S. Plagenhoef, F. G. Evans, and T. Abdelnour, "Anatomical data for analyzing human motion," Research Quarterly for Exercise and Sport, vol. 54, no. 2, pp. 169-178, 1983.

[16] W. Erdmann, "Geometry and inertia of the human body - review of research," Acta of Bioengineering and Biomechanics, vol. 1, no. 1, pp. 23-35, 1999.

[17] H. Zhao, E. Ambrose, and A. D. Ames, "Preliminary results on energy efficient 3D prosthetic walking with a powered compliant transfemoral prosthesis," in Robotics and Automation (ICRA), 2017 IEEE International Conference on. IEEE, 2017, pp. 1140-1147.

[18] R. M. Murray, S. S. Sastry, and L. Zexiang, A Mathematical Introduction to Robotic Manipulation, 1st ed. CRC Press, Inc., 1994.

[19] R. W. Sinnet, M. J. Powell, R. P. Shah, and A. D. Ames, "A humaninspired hybrid control approach to bipedal robotic walking," IFAC Proceedings Volumes, vol. 44, no. 1, pp. 6904 - 6911, 2011, 18th IFAC World Congress.

[20] R. Gehlhar, Y. Chen, and A. D. Ames, "Data-driven characterization of human interaction for model-based control of powered prostheses," arXiv preprint arXiv:2003.07524v1, 2020. 